\title{
Subtypes of oligodendroglioma defined by 1p,19q deletions, differ in the proportion of apoptotic cells but not in replication-licensed non-proliferating cells
}

\author{
S. B. Wharton • E. Maltby $\cdot$ D. A. Jellinek $\cdot$ D. Levy $\cdot$ \\ N. Atkey $\cdot$ S. Hibberd $\cdot$ D. Crimmins $\cdot$ K. Stoeber • \\ G. H. Williams
}

Received: 21 September 2006 / Revised: 16 November 2006 / Accepted: 20 November 2006 / Published online: 9 December 2006

(C) Springer-Verlag 2006

\begin{abstract}
Oligodendrogliomas may be divided into those with deletion of chromosomes $1 p$ and $19 q$ (Del+), and those without (Del-). Del+ tumours show better survival and chemoresponsiveness but the reason for this difference is unknown. We have investigated whether these subgroups differ in (a) apoptotic index, (b) the proportion of cells licensed for DNA replication but not in-cycle, and (c) the relative length of $\mathrm{G}_{1}$-phase. Fluorescence in situ hybridisation with probes to $1 \mathrm{p}$ and $19 \mathrm{q}$ was used to determine the deletion
\end{abstract}

S. B. Wharton · S. Hibberd

Department of Histopathology, Sheffield Teaching Hospitals, Sheffield, UK

S. B. Wharton $(\varangle)$

Academic Unit of Pathology, University of Sheffield, Medical School, Beech Hill Road, Sheffield, S10 2RX, UK

e-mail: s.wharton@sheffield.ac.uk

E. Maltby $\cdot$ N. Atkey

Department of Cytogenetics, Sheffield Children's Hospital, Sheffield, UK

D. A. Jellinek

Department of Neurosurgery, Sheffield Teaching Hospitals, Sheffield, UK

D. Levy

Department of Clinical Oncology,

Weston Park Hospital, Sheffield, UK

D. Crimmins

Department of Paediatric Neurosurgery,

Leeds General Infirmary, Leeds, UK

K. Stoeber · G. H. Williams

Department of Pathology, Royal Free and University College

Medical School, University College London, London, UK status of 54 oligodendrogliomas, including WHO grades II and III. The apoptotic index was determined using counts of apoptotic bodies. Replication-licensed non-proliferating cells were determined from the Mcm2 minus Ki67 labelling index, whilst the geminin to Ki67 ratio was used as a measure of the relative length of $\mathrm{G}_{1}$. Del+ oligodendrogliomas showed a higher apoptotic index than Del- tumours $(P=0.037)$; this was not accounted for by differences in tumour grade or in proliferation. There were no differences in the Mcm2 - Ki67 index or in the geminin/Ki67 ratio between the subgroups, but grade III tumours showed a higher proportion of licensed non-proliferating cells than grade II tumours $(P=0.001)$. An increased susceptibility to apoptosis in oligodendrogliomas with $1 \mathrm{p} \pm 19 \mathrm{q}$ deletion may be important in their improved clinical outcome compared to Del- tumours.

Keywords Oligodendroglioma - Apoptosis · Replication $\cdot$ DNA replication licensing $\cdot$ Cytogenetic

\section{Introduction}

Oligodendrogliomas are tumours composed of cells that morphologically resemble oligodendrocytes and that range in spectrum from well differentiated to anaplastic tumours. The current WHO classification of CNS tumours recognises two histological grades, WHO grades II and III, and grading is a significant predictor of survival [23]. Oligodendrogliomas have generally been considered to have a better prognosis than the corresponding grades of diffuse astrocytoma, and recent data continues to support this [21]. However, oligodendrogliomas, like astrocytomas, are diffusely 
infiltrating tumours, precluding complete surgical resection, and they may show malignant progression over time. There is therefore a role for adjuvant radiotherapy and/or chemotherapy, particularly for anaplastic tumours.

Approximately two-thirds of oligodendrogliomas show allelic losses from chromosomes $1 p$ and $19 q[24$, 25], a genetic profile that appears to be associated with clinical outcome. Some anaplastic oligodendrogliomas respond to combination chemotherapy [2,3], and better progression free survival and chemo-responsiveness appear to be linked to the presence of these deletions $[4,34]$. Results from two recent large-scale trials provide support for the view that the presence of $1 p 19 q$ deletion identifies a subgroup with better clinical outcome $[1,41] .1 p 19 q$ deletion may also identify better prognosis in low-grade oligodendrogliomas [13].

There may be some histopathological differences between the two genetic subgroups. Classical oligodendroglioma features, such as perinuclear haloes (in $>50 \%$ of cells), uniform rounded nuclei, chickenwire vascular pattern and calcification, are associated with 1p 19q loss [14, 27, 42], suggesting that classical features, such as perinuclear haloes associated with tumour cellularity, can predict genotype [14]. However, there are cases of histologically typical oligodendrogliomas without $1 \mathrm{p} 19 \mathrm{q}$ deletion and more astrocytic appearing tumours with deletion. For these cases clinical behaviour appears to be better predicted by genotype than phenotype, so that $1 \mathrm{p} 19 \mathrm{q}$ deletion provides additional information to conventional histopathological analysis [27]. Some studies suggest that oligodendrogliomas with $1 \mathrm{p} 19 \mathrm{q}$ deletion have lower activity of $O^{6}$-methylguanine DNA methyltransferase (MGMT) due to promoter hypermethylation and decreased expression. MGMT is a DNA repair enzyme associated with resistance to alkylating agents, so this may contribute to chemosensitivity [18], although other studies have not found a clear association [42]. At present, therefore, the biological basis for the intrinsically better prognosis of the $1 p 19 q$ deletion subgroup and the possible basis of a different interaction with adjuvant therapies remain poorly understood.

In this study we hypothesise that the difference in behaviour of tumours with and without these deletions is due to differences in kinetic properties of the tumours related to proliferation and apoptosis. Apoptotic bodies are often a conspicuous feature in oligodendrogliomas and levels of apoptosis increase with grade [43]. Tumour growth depends on the net effects of cell proliferation and loss [35] so that a subgroup of tumours with a greater propensity to apoptosis versus proliferation may have slower growth. It is thus possible that increased susceptibility of tumour cells to undergo apoptosis might underlie both a better intrinsic biological behaviour and a greater sensitivity to adjuvant therapy. We hypothesise therefore that tumours with $1 \mathrm{p}, 19 \mathrm{q}$ deletions show a greater tendency to apoptosis that is reflected in increased basal levels of apoptosis in the tumours.

Differences in kinetic parameters related to DNA replication licensing are also candidates for relevance to growth capacity and chemosensitivity. The initiation of chromosomal replication is a crucial decision point in cell proliferation that lies at the point of convergence of all oncogenic signalling and transduction pathways that trigger proliferation. DNA replication initiation is precisely controlled through a core set of licensing factors (Orc1-6, Cdc6, Cdt1, Mcm2-7) that sequentially assemble into pre-replicative complexes (pre-RCs) at $\sim 30,000$ replication origins scattered along the chromosomes, resulting in chromatin being 'licensed' for replication in the subsequent $\mathrm{S}$ phase. Licensed replication origins are activated by the concerted action of $\mathrm{S}$ phase promoting kinases $(\mathrm{Cdk} 2)$ and the ASK-dependent $\mathrm{Cdc7}$ kinase in a process commonly known as 'origin firing'. Origin firing results in local melting of the DNA helix at replication origins and the recruitment of DNA polymerases (reviewed in [40]). Notably the Mcm2-7 replication licensing factors, which have been shown to act as a replicative DNA helicase, are present within the nucleus in cells in-cycle, but are down-regulated in out-of-cycle states $[10,38]$.

We have previously shown that higher grade oligodendrogliomas show a greater proportion of cells that are licensed for DNA replication, as determined by the $\mathrm{Mcm} 2$ labelling index, which is of prognostic significance [44]. Certain tissues contain a population of licensed but non-proliferating cells $[10,38]$ and higher labelling indices for $\mathrm{Mcm} 2$ than for the conventional proliferation marker Ki67 (which marks all four phases of the cell-cycle) suggest that this may be true for oligodendrogliomas [44]. A population of licensed but nonproliferating cells might conceivably resist cell-cycle dependent cytotoxicity and form a viable cell cache for tumour re-population. We have used the difference between the Mcm2 and Ki67 labelling indices $(\mathrm{Mcm} 2-\mathrm{Ki} 67 \mathrm{LI})$ as an assay of this population.

It is essential for faithful propagation of the genome that origins are fired once and only once in each cell cycle to avoid re-replication events, which would lead to gene amplification. Geminin, a $23.5 \mathrm{kDa}$ nuclear protein, is an endogenous regulator of chromosomal replication, absent during G1 phase but allowed to 
accumulate during S, G2 and M phase [17]. Geminin acts to prevent a second, inappropriate firing of replication origins by competitively binding to Cdt1 and thereby blocking recruitment of the Mcm2-7 helicase to replication origins $[39,48]$. Thus pre-RC re-assembly and firing are prevented. At the metaphase to anaphase transition in mitosis, the anaphase promoting complex $(\mathrm{APC} / \mathrm{C})$ becomes activated and geminin is targeted for polyubiquitination and degradation via the $26 \mathrm{~S}$ proteosome pathway, lowering levels to the point where the protein no longer interferes with assembly of pre-RCs during the following G1 phase [17]. Since geminin is present in $\mathrm{S}-\mathrm{G} 2-\mathrm{M}$ phase, the ratio of geminin to Ki67 may provide an index of the relative length of $G_{1}$-phase. We have previously shown that this ratio decreases in higher-grade oligodendrogliomas, which may be a reflection of a shortened $\mathrm{G}_{1}$ in anaplastic tumours [45]. The length of $\mathrm{G}_{1}$-phase may be relevant to chemotherapeutic agents targeting the cell cycle machinery. We have therefore determined whether cytogenetic subgroups of oligodendrogliomas differ in their geminin/Ki67 ratio.

\section{Materials and methods}

A total of 55 cases of oligodendroglioma were identified and paraffin blocks retrieved from the archives of the Histopathology Department of the Royal Hallamshire Hospital, Sheffield, from a 12 year period from 1985 to 1997. As previously described [45], 25 cases were graded as WHO grade II (mean age 37.4 years) and 30 as grade III (mean age 44.6 years). The series comprised 22 female and 33 male patients. All of the cases were resection specimens; stereotactic and other small biopsies were excluded. Of these cases, 47 were first resections. Eight cases were recurrent resections that fell within the study period; the initial resections on these cases were prior to the study period and not included. All of the cases were used for the labelling index analyses but only the 47 first resections were used for survival analysis. Clinical follow-up data and data on radiotherapy and chemotherapy were obtained from the Clinical Oncology Department at Western Park Hospital, Sheffield. Approval for the study was granted by the Local Research Ethics Committee.

\section{Immunohistochemistry}

Immunohistochemistry was performed on serial sections from a representative paraffin block from each case using a standard $\mathrm{ABC}$ method and the signal visualised using diaminobenzidine. Immunostaining was performed to Ki67 (Novocastra), Mcm2 (BD Transduction Laboratories) and geminin (G95, rabbit polyclonal) on this series of tumours as previously described [45]. Apoptotic bodies were counted on haematoxylin and eosin stained sections. Areas of highest cellularity were used for quantitation. The percentage of labelled cells, derived from a count of at least 1000 cells, was determined for each marker using an eyepiece graticule [44, 45].

\section{Fluorescence in situ hybridisation}

Fluorescence in situ hybridisation (FISH) was performed on $4 \mu$ thick sections using the technique previously described [7]. The Vysis dual colour probe sets LSI 1p36/LSI 1q25 and LSI 19p13/LSI 19q13 were used according manufacturers instructions; probes were hybridised overnight using a PTC Peltier Thermal Cycler. Analysis was performed independently by two scientists, both scoring 30 cells for each probe set.

\section{Statistical analyses}

Statistical analyses were performed using the statistical package for the social sciences (SPSS v10.1). Comparison of age was made using an unpaired $t$ test. Data on labelling indices was either not normally distributed and/or did not satisfy criteria for equality of variance between groups (Levene's test). Therefore comparisons of labelling indices between groups were performed using the non-parametric Mann-Whitney $U$ test. Fisher's exact test was used for assessment of distribution between groups. For comparison of survival between groups, Kaplan Meier plots were constructed and the log rank test used. Multivariable linear regression analysis was used to examine the relationship of apoptotic index (AI) to proliferation and deletion status. Ki67 labelling index (LI) was used as measure of the former, rather than Mcm2 LI, as the latter also includes replication licensed but nonproliferating cells. To model a difference in the slope of the relationship between $\mathrm{AI}$ and Ki67 LI in the two subgroups, an interactive term between Ki67 LI and deletion status was included [Model: $\mathrm{AI}=\beta_{0}+\left(\beta_{1} \times \mathrm{Ki} 67 \mathrm{LI}\right)+$ $\left(\beta_{2} \times\right.$ deletion status $)+\left(\beta_{3} \times\right.$ Ki67 LI $\times$ deletion status $\left.)\right]$.

\section{Results}

Characteristics of the deletion sub-groups

FISH analysis for $1 p$ and $19 q$ deletion was successfully performed in 54 out of 55 cases (Fig. 1). Cases were 
Fig. 1 Representative FISH images using the VYSIS dual colour probe sets; a relative loss of 1p (red) to 1q (green) with probable increase of chromosome number, b simple deletion of $1 \mathrm{p}(\mathrm{red})$, c relative loss of $19 \mathrm{q}(\mathrm{red})$ to $19 \mathrm{p}$ (green) with probable increase of chromosome number, $\mathbf{d}$ simple deletion of $19 \mathrm{q}($ red $)$
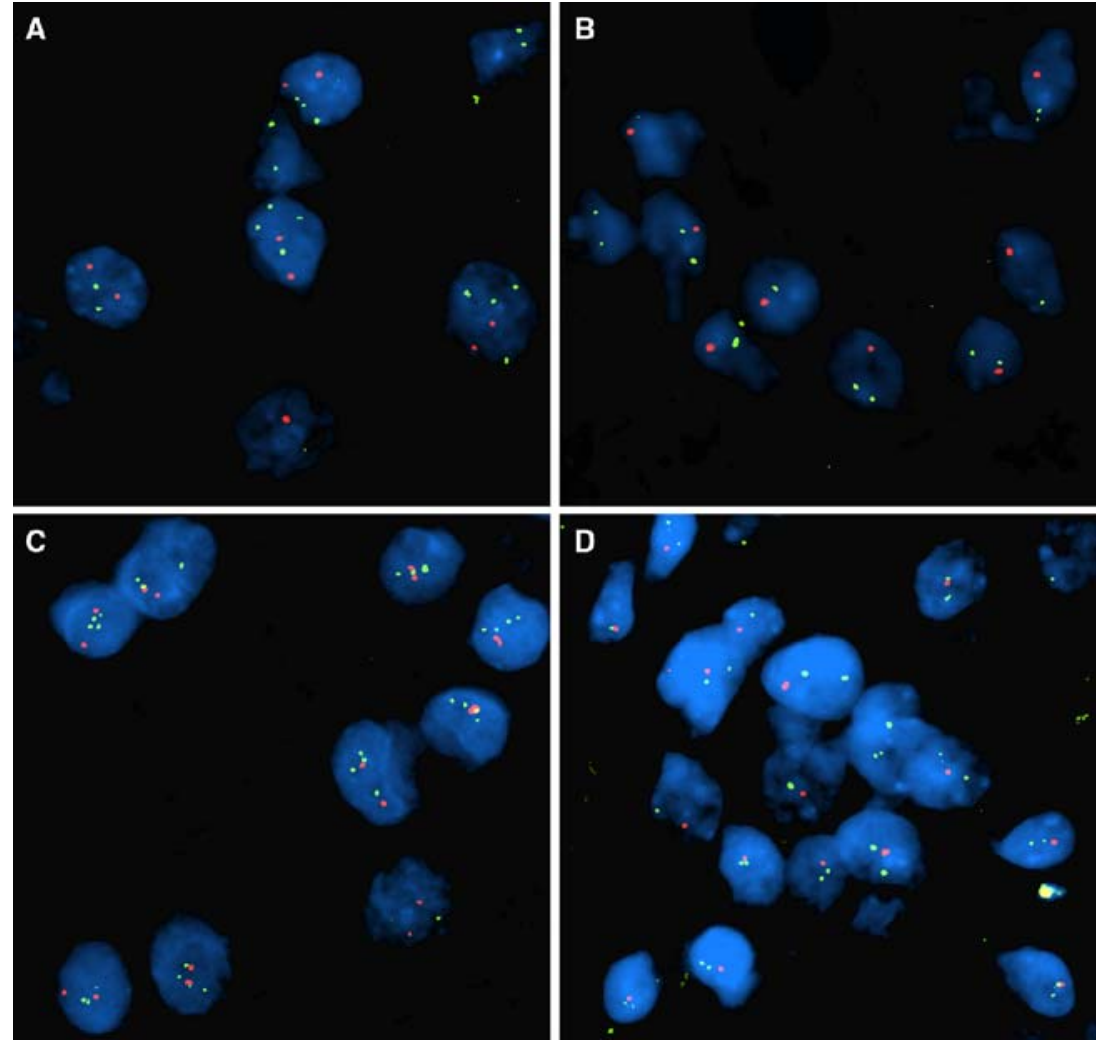

assigned to the Del+ group if they had deletion of both $1 \mathrm{p}$ and $19 \mathrm{q}$, or $1 \mathrm{p}$ alone. Cases were assigned to the Del- group if they had no deletion or deletion of $19 q$ only. There were $34 \mathrm{Del}+$ cases and 20 Del- (Table 1). The Del+ group had an older mean age than the Delgroup (Table 2) $(P=0.002)$. There was no difference in the distribution of histological grade between the two groups $(P=1)$.

\section{Survival analysis}

Survival for the 47 cases included in the survival analysis was taken from the date of the surgical resection. The eight (recurrent) cases excluded from this analysis were distributed evenly between Del+ and Delgroups. The Del+ group showed a trend to better survival (Fig. 2) but this did not reach significance $(P=0.092)$, probably due to the number of censored

Table 1 Results of FISH analysis

\begin{tabular}{llrll}
\hline Group & Deletion & Number & $\begin{array}{l}\text { No. in } \\
\text { Del group }\end{array}$ & $\begin{array}{l}\% \text { of } \\
\text { cases }\end{array}$ \\
\hline Del+ & $1 \mathrm{p} \mathrm{19q}$ & 34 & 34 & 63 \\
Del- & $1 \mathrm{p}$ & 0 & & \\
& None & 17 & 20 & 37 \\
& $19 \mathrm{q}$ & 3 & & \\
\hline
\end{tabular}

Table 2 Charateristics of cytogenetic sub-groups

\begin{tabular}{lll}
\hline Group & Del+ & Del- \\
\hline Mean age, SD & $46.0,11.5$ & $33.1,17.9$ \\
Grade II & 16 & 9 \\
Grade III & 18 & 11 \\
$\%$ of grade II & 47.1 & 45.0 \\
\hline
\end{tabular}

cases. Further survival analysis to include multivariate parameters was therefore not attempted.

Comparison of indices between cytogenetic subgroups

The labelling index for apoptotic bodies (AI), assessed from $H \& E$ stained sections was used as a measure of apoptosis. Higher levels of apoptosis were seen in the Del+ group, carrying either deletions of $1 \mathrm{p}$ and $19 \mathrm{q}$, or $1 \mathrm{p}$ alone $(P=0.037)$ (Table 3, Fig. 3$)$. To further analyse the difference in apoptosis between the two cytogenetic subgroups, we considered whether higher levels of apoptosis in the Del+ group were a reflection of higher cell turnover. However, there was no difference in replication-licensed or proliferating cells between the two subgroups as assessed by $\mathrm{Mcm} 2$ $(P=0.87)$ or Ki67 $(P=0.77)$, respectively. Inspection of scatterplots of AI against Ki67 labelling index for the two subgroups suggested that with increasing 


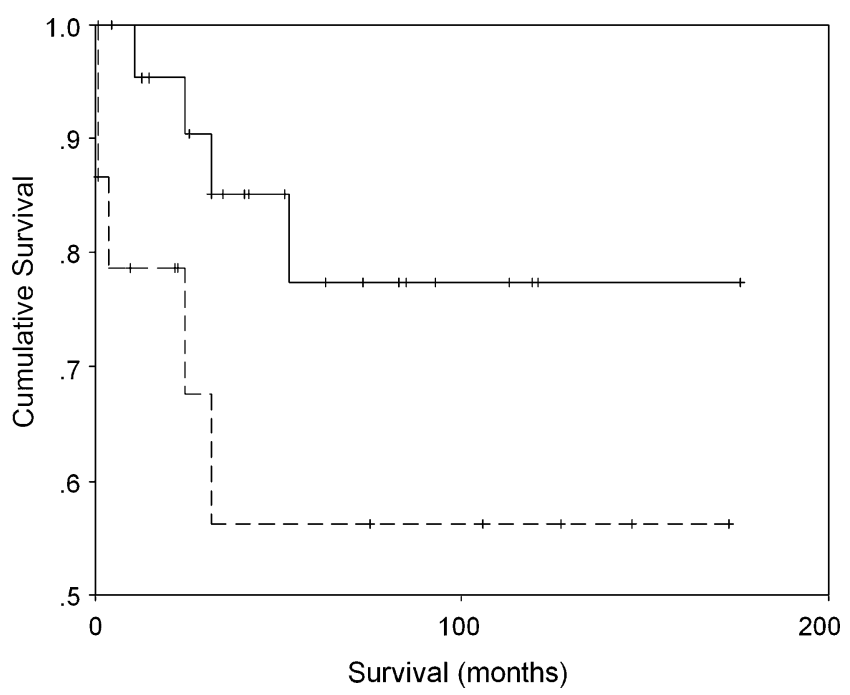

Fig. 2 Kaplan Meier survival curves for Del+ (solid line) and Del- (dashed line) oligodendroglioma subgroups. Censored cases shown as cross marks

Table 3 Descriptive data for kinetic measures

\begin{tabular}{lllrl}
\hline Subgroup & Measure & AI & Mcm2 - Ki67 & Gem/Ki67 \\
\hline Del- & Mean (SD) & $0.72(0.38)$ & $8.31(22.21)$ & $0.39(0.25)$ \\
& Median (IQR) & $0.60(0.48)$ & $6.65(23.18)$ & $0.38(0.44)$ \\
Del+ & Mean (SD) & $1.10(0.69)$ & $12.51(15.11)$ & $0.29(0.15)$ \\
& Median (IQR) & $0.95(1.00)$ & $7.15(21.88)$ & $0.26(0.22)$
\end{tabular}

$A I$ apoptotic index, $M c m 2-K i 67 \mathrm{Mcm} 2$ minus Ki67 labelling index, Gem/Ki67 ratio of geminin to Ki67 indices, $S D$ standard deviation, $I Q R$ interquartile range

tumour proliferation, apoptosis shows a greater increase in the Del+ subgroup (Fig. 4). Because this suggested a steeper relationship of AI to proliferation in the Del+ subgroup, multivariable regression analysis was carried out with a model, incorporating Ki67 LI, deletion status and a term for interaction between these two variables as predictors of apoptosis index. This gave an overall significance of $P=0.002$ for the model. The interaction between Ki67 LI and deletion status was significant $(P=0.031)$ and AI rose more steeply against Ki67 LI in the Del+ group (AI $\alpha$ 0.58xKi67LI) than in the Del-group (AI $\alpha 0.13$ Ki67 LI).

The difference between Mcm2 and Ki67 labelling indices $(\mathrm{Mcm} 2-\mathrm{Ki} 67 \mathrm{LI})$ was used as a measure of the fraction of tumour cells that were licensed but not proliferating. In general tumours showed a higher labelling index for Mcm2 than Ki67, indicating the presence of a population of licensed cells that are not actively replicating. The $\mathrm{Mcm} 2-\mathrm{Ki} 67 \mathrm{LI}$ was higher in grade III than in grade II tumours $(P=0.001$, Fig. 5$)$. However, we demonstrated no difference in the proportion of licensed, non-cycling cells by this method

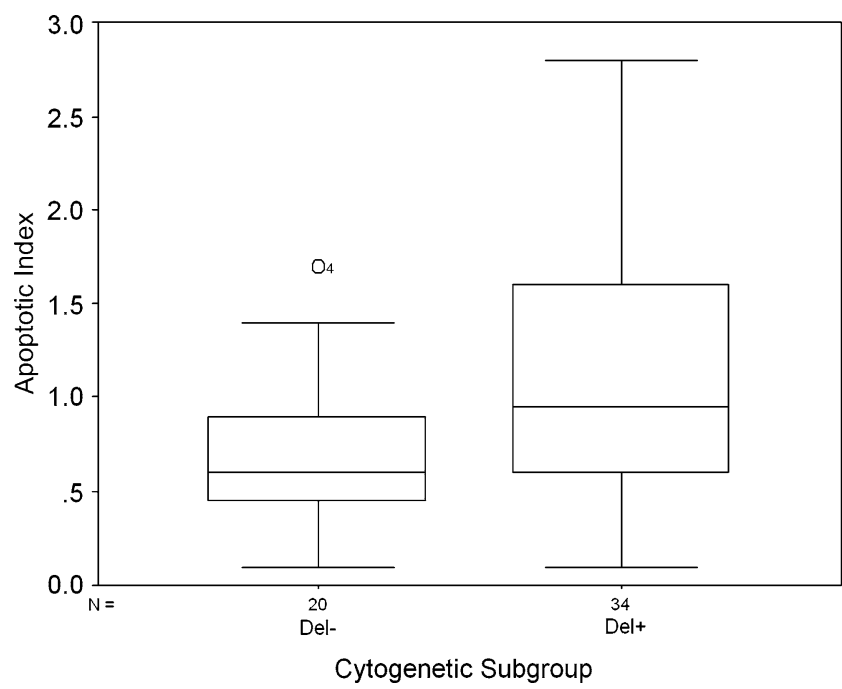

Fig. 3 Boxplot showing apoptotic indices in Del- and Del+ subgroups

between the two cytogenetic subgroups of oligodendrogliomas $(P=0.46)$. We also demonstrated no difference in the geminin/Ki67 ratio $(P=0.22)$.

\section{Discussion}

The basis for the difference in clinical behaviour between oligodendrogliomas with deletions of $1 \mathrm{p}$ and $19 q(\mathrm{Del}+)$ and those without (Del-) remains unclear, but its elucidation may yield important new avenues for improved therapeutic approaches. In this study we have investigated whether there are differences in parameters related to tumour cell proliferation and death in these two cytogenetic subgroups of tumours.

The tumours were typed using FISH with probes to $1 \mathrm{p}$ and $19 \mathrm{q}$. In our series, $63 \%$ of cases showed deletions in $1 \mathrm{p}$ and $19 \mathrm{q}$, or $1 \mathrm{p}$ alone, a similar proportion to that reported in series of oligodendrogliomas in the literature where figures for combined loss from approximately 50 to $70 \%$ of cases have been described (sometimes higher in classical appearing WHO grade II tumours) [13, 19, 24, 25, 33]. Del+ and Del- had a similar distribution of histological grades whilst the older average age in the Del+ group is also unlikely to be of relevance to our findings as age is in general associated with a worse clinical behaviour, and opposite to the effects here. A trend towards better overall survival in Del+ cases in our series did not reach significance, probably due to the number of total and censored cases.

Analysis of the FISH probes in this cohort of cases revealed a pattern of multiple signals in some cases, indicating that increased copy number of chromosomes 1 and 19 were present, but that there was still effective loss 
Fig. 4 Scatterplots of apoptosis index $(A I)$ versus Ki67 labelling index for Del- (left) and Del+ (right) cases
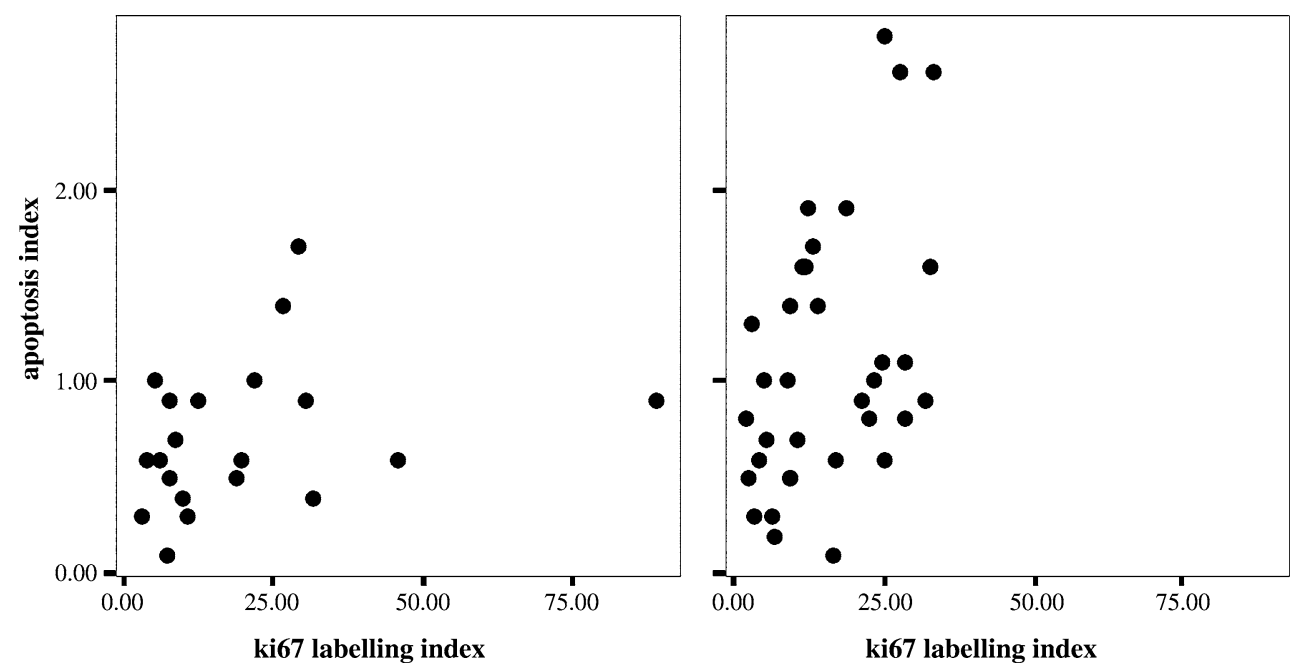

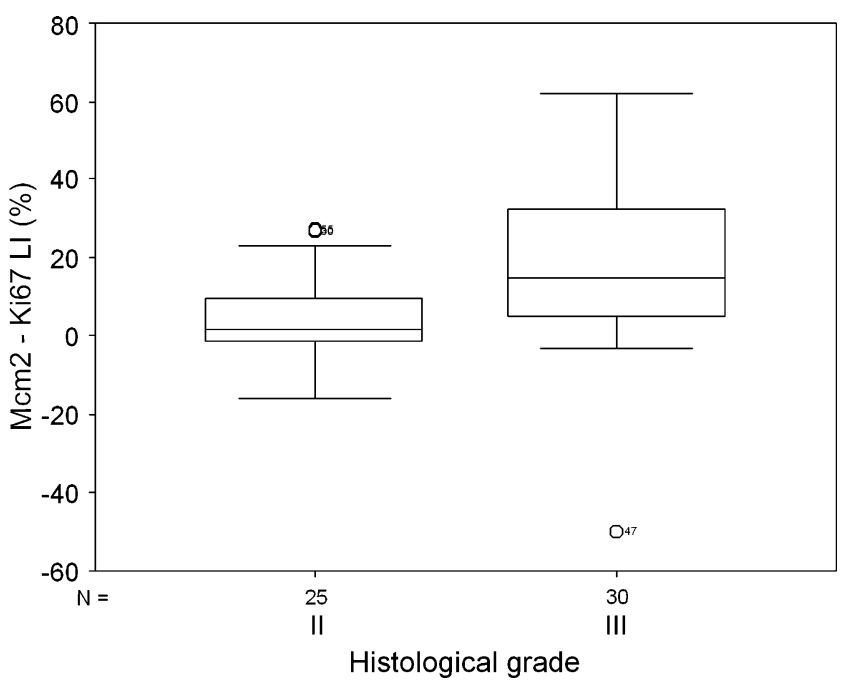

Fig. 5 Boxplot of Mcm2 minus Ki67 labelling index according to histological grade

of heterozygosity of short arm chromosome 1 and long arm chromosome 19. (Fig. 1) These cases were interpreted Del+. One other subgroup was identified in the cohort: three cases in which there was $19 \mathrm{q}$ deletion present without concomitant $1 \mathrm{p}$ deletion. There was no significant association of these subgroups in our series with an obvious pathological feature, but more cases should be studied to determine any clinico-pathological correlation. The multiple signal cases, which would indicate complex aneuploid karyotypes, usually found in tumours with more malignant status, may be of particular interest. One other group has identified the single 19q deletion status cases and have suggested that $19 \mathrm{q}$ deletion alone may also predict a favourable prognosis [22].

We have previously reported values for apoptosis, $\mathrm{Mcm} 2$, Ki67 and geminin in this series of tumours
[45], but we now define indices and relate these to cytogenetic subtype. We have investigated three selected parameters that we hypothesise may affect survival and therapeutic response. Apoptosis is an important process in tumour biology as defective apoptosis induction may contribute to cancer development and the susceptibility of tumour cells to apoptosis may affect rate of growth. Apoptosis may be induced by adjuvant therapies and is likely to become an increasingly important molecular therapeutic target as specific pathways are defined (reviewed in [26]). Apoptosis is variable between tumour types and a number of factors may contribute to apoptosis induction in a tumour. The apoptotic index often appears to be a function of the proliferation rate, with higher levels in more rapidly cycling tumours. In oligodendrogliomas, we and others have previously shown that apoptosis tends to increase with proliferation and with tumour grade $[29,43]$, though it has not been shown to be an independent prognostic factor. A similar relationship of apoptosis to histological grade and proliferation has also been demonstrated in the diffuse astrocytomas [8]. Although quantification of apoptosis, and so comparison between tumour types and studies, is beset with methodological difficulties, there is evidence that oligodendrogliomas show higher levels of apoptosis than other brain tumours of comparative WHO grade [15, 28]. Alterations in regulatory factors related to molecular genetic changes are likely to be important in setting the susceptibility to apoptosis in a given tumour type, whilst other factors such as ischaemia and the influence of tumoural inflammatory cells may also contribute to apoptosis induction. Thus variation in apoptosis is a candidate to explain differences in clinical behaviour of tumour subtypes. 
In this series of oligodendrogliomas we have assessed apoptosis using counts of apoptotic bodies. This method has been used for other tumour types, including astrocytomas [8]. In a previous oligodendroglioma series we showed that the apoptotic index correlates with an assessment of apoptosis using the TUNEL method [43], but it is a much simpler method. In addition, the TUNEL technique can be capricious and lacks specificity as it may detect DNA damage in cells from processes other than apoptosis, including necrosis [12]. Immunohistochemical detection of caspase 3 has also been used to detect apoptotic cells in histological sections [5]. We have previously carried out caspase 3 immunohistochemistry on this series [45], but this showed weaker relationships to grade and proliferation than apoptotic index using counts of apoptotic bodies. Therefore, for this study, apoptotic index based on counts of apoptotic bodies was selected a priori for analysis.

We have demonstrated that Del+ oligodendrogliomas show a higher apoptotic index than Del- tumours, supporting the hypothesis that tumour cells from Del+ cases may have a greater susceptibility to undergo apoptosis. We have not investigated the molecular pathways that might lead to this difference. However, it is not simply a reflection of increased cell turnover as Del+ cases show no increase in either Mcm2 or Ki67 labelling indices compared to Del- cases (although a difference in cell-cycle length is not formally excluded). The greater rate of increase in apoptotic index against Ki67 labelling index for Del+ than Del- tumours suggests that oligodendrogliomas with $1 \mathrm{p}, 19 \mathrm{q}$ deletion have a greater tendency to the induction of apoptosis at higher rates of proliferation.

Immunohistochemical demonstration and quantification of components of the DNA replication licensing machinery have recently been used for prognostic [16, $30,32,44]$ and surveillance [37, 46, 47] studies in human tumours. Theoretically demonstration of MCM proteins may be considered superior to Ki67 as it identifies not only cycling cells, but also those non-cycling cells with proliferative potential $[10,38]$, and this superiority is born out in many of these studies. Combined assessment of these markers with other cell cycle related markers may be used to obtain information on the relative length of cell cycle phases in archival biopsy material $[9,20,31]$. In this study, which adds to these applications, we have used a variable generated from the difference between the $\mathrm{Mcm} 2$ and Ki67 labelling indices (Mcm2-Ki67 LI) for each case to assess the proportion of tumour cells that are licensed to replicate but not actually in cycle. This index has been previously used in renal cell cancer, where it increases with tumour grade [6]. We now also demonstrate that this index increases with tumour grade in oligodendrogliomas, suggesting that the pool of cells that are licensed but not proliferating is larger in more anaplastic tumours, evidence for greater dysregulation of the origin licensing pathway with anaplasia. Such a licensed, non-proliferating fraction might conceivably act as a treatment-resistant population that could re-grow after adjuvant therapy. There is increasing evidence for tumour stem cells in brain tumours (reviewed in [11]) and it is intriguing to speculate whether a stem cell population, responsible for perpetuating tumour growth, might reside in this fraction. Its assay is therefore of potential interest and we hypothesised that the size of this fraction might be greater in Del- tumours. However, we did not demonstrate any difference in Mcm2 - Ki67 LI between Del+ and Del- tumours, suggesting that this is not important in determining their clinical differences.

We have previously shown that the ratio of geminin to Ki67 labelling indices is increased in grade III versus grade II oligodendrogliomas [45], implying a shortened $\mathrm{G}_{1}$-phase in higher grade tumours (assuming that other cell cycle phases are not substantially altered in duration). In this study, however, we have not demonstrated evidence for a differing $G_{1}$-phase duration between Del+ and Del- tumours.

Molecular differences between these two cytogenetic subtypes of oligodendroglioma are beginning to be defined, which will allow therapeutically relevant molecular classification [24]. Some of these genetic differences likely relate to the regulation of apoptosis, and may differ in the two subgroups. This study has shown that those oligodendrogliomas with $1 p \pm 19 q$ deletions have higher apoptotic indices than those without. Differences in cell death susceptibility may affect prognosis through a treatment independent effect on intrinsic growth rate. But apoptosis may also be induced by adjuvant therapies, and amongst the gliomas this may be particularly important for oligodendroglial tumours [36]. This study does not allow distinction between intrinsic growth rate and treatment response contributions of apoptosis. It has also been limited to histological methods and has not provided functional data on susceptibility or defined its molecular basis. However, the findings support the hypothesis that differences in clinical outcomes between these cytogenetic subgroups is related to differing susceptibility to apoptosis induction and suggest that detailed comparative studies of apoptotic pathways in molecular subgroups of oligodendrogliomas are warranted.

Acknowledgments This work received support from the Sheffield Brain Tumour Research Fund and the Evie Clarke Fund. 


\section{References}

1. Cairncross G, Berkey B, Shaw E, Jenkins R, Scheithauer B, Brachman D, Buckner J, Fink F, Souhami L, Laperierre N, Mehta M, Curran W (2006) Phase III trial of chemotherapy plus radiotherapy compared with radiotherapy alone for pure and mixed anaplastic oligodendroglioma: intergroup radiation therapy oncology group trial 9402. J Clin Oncol 24(18):2707-2714

2. Cairncross J, MacDonald D (1988) Successful chemotherapy for recurrent malignant oligodendroglioma. Ann Neurol 23:360-364

3. Cairncross J, MacDonald D (1991) Chemotherapy for oligodendroglioma. Arch Neurol 48:225-227

4. Cairncross J, Ueki K, Zlatescu M, Lisle D, Finkelstein D, Hammond R, Silver J, Stark P, MacDonald D, Ino Y, Ramsay D, Louis D (1998) Specific genetic predictors of chemotherapeutic response and survival in patients with anaplastic oligodendrogliomas. J Natl Cancer Inst 90(19):1473-1479

5. Duan W, Garner D, Williams S, Funckes-Shippy C, Spath I, Blomme E (2003) Comparison of immunohistochemistry for activated caspase- 3 and cleaved cytokeratin 18 with the TUNEL method for quantification of apoptosis in histological sections of PC-3 subcutaneous xenografts. J Pathol 199:221-228

6. Dudderidge T, Stoeber K, Loddo M, Atkinson G, Fanshawe T, Griffiths D, Williams G (2005) Mcm2, Geminin, and Ki67 define proliferative state and are prognostic markers in renal cell carcinoma. Clin Cancer Res 11:2510-2517

7. Ellis C, Dyson M, Stephenson T, Maltby E (2005) HER2 amplification status in breast cancer: a comparison between immunohistochemical staining and fluorescence in situ hybridisation using manual and automated quantitative image analysis scoring techniques. J Clin Pathol 58:710-714

8. Ellison D, Steart P, Gatter K, Weller R (1995) Apoptosis in cerebral astrocytic tumours and its relationship to expression of the bcl-2 and p53 proteins. Neuropathol Appl Neurobiol 21:352-361

9. Endl E, Kausch I, Baack M, Knippers R, Gerdes J, Scholzen T (2001) The expression of Ki-67, MCM3, and p27 defines distinct subsets of proliferating, resting, and differentiated cells. J Pathol 195:457-462

10. Eward K, Obermann E, Shreeram S, Loddo M, Fanshawe T, Williams C, Jung H, Prevost A, Blow J, Stoeber K, Williams G (2004) DNA replication licensing in somatic and germ cells. J Cell Sci 117:5875-5886

11. Galderisi U, Cipollaro M, Giordano A (2006) Stem cells and brain cancer. Cell Death Differ 13:5-11

12. Grasl-Kraupp B, Ruttkay-Nedecky B, Koudelka H, Bukowska K, Bursch W, Schulte-Hermann R (1995) In situ detection of fragmented DNA (TUNEL assay) fails to discriminate among apoptosis, necrosis and autolytic cell death: a cautionary note. Hepatology 21:1465-1468

13. Kanner A, Staugitis S, Castilla E, Chernova O, Prayson R, Vogelbaum M, Stevens G, Peereboom D, Suh J, Lee S-Y, Tubbs R, Barnett G (2006) The impact of genotype on outcome in oligodendroglioma: validation of the loss of chromosome arm $1 \mathrm{p}$ as an important factor in clinical decision making. J Neurosurg 104:542-550

14. Kim S, Kim H, Kim T (2005) Clinical, histological, and immunohistochemical features predicting $1 \mathrm{p} / 19 \mathrm{q}$ loss of heterozygosity in oligodendroglial tumors. Acta Neuropathol (Berl) 110:27-38

15. Leaver H, Whittle I, Wharton S, Ironside J (1998) Apoptosis in human primary brain tumours. Br J Neurosurg 12:539-546

16. Li S, Xue W, Khoo U, Ngan H, Chan K, Tam I, Chiu P, Ip P, Tam K, Cheung A (2005) Replicative MCM7 protein as a proliferation marker in endometrial carcinoma: a tissue microarray and clinicopathological analysis. Histopathology 46:307-313

17. McGarry T, Kirschner M (1998) Geminin, an inhhibitor of DNA replication, is degraded during mitosis. Cell 93:1043-1053

18. Mollemann M, Wolter M, Felsberg J, Collins V, Reifenberger $\mathrm{G}$ (2005) Frequent promoter hypermethylation and low expression of the MGMT gene in oligodendroglial tumors. Int J Cancer 113:379-385

19. Nigro J, Takahashi $M$, Ginzinger $D$, Law $M$, Sandra $P$, Jenkins R, Aldape K (2001) Detection of 1p and 19q loss in oligodendroglioma by quantitative microsatellite analysis, a real-time quantitative polymerase chain reaction assay. Am J Pathol 158:1253-1262

20. Obermann E, Eward K, Dogan A, Paul E, Loddo M, Munson P, Williams G, Stoeber K (2005) DNA replication licensing in peripheral B-cell lymphoma. J Pathol 205:318-328

21. Ohgaki H, Kleihues P (2005) Population-based studies in incidence, survival rates and genetic alterations in astrocytic and oligodendroglial gliomas. J Neuropathol Exp Neurol 64:479-489

22. Perry A, Fuller C, Banerjee R, Brat D, Scheithauer B (2003) Ancillary FISH analysis for $1 \mathrm{p}$ and $19 \mathrm{q}$ status: preliminary observations in 287 gliomas and oligodendroglioma mimics. Front Bioscience 8:a1-a9

23. Reifenberger G, Kros J, Burger P, Louis D, Collins V (2000) Oligodendroglioma. In: Kleihues P, Cavenee W (eds) Pathology and Genetics. Tumours of the Nervous System, IARC Press, Lyon, pp 56-64

24. Reifenberger G, Louis D (2003) Oligodendroglioma: toward molecular definitions in diagnostic neuro-oncology. J Neuropathol Exp Neurol 62:111-126

25. Reifenberger J, Reifenberger G, Liu L, James C, Wechsler W, Collins V (1994) Molecular genetic analysis of oligodendroglial tumors show preferential allelic deletions on 19q and 1p. Am J Pathol 145:1175-1190

26. Russo A, Terrasi M, Agnese V, Santini D, Bazan V (2006) Apoptosis: a relevant tool for anticancer therapy. Ann Oncol 17(Suppl 7):vii115-vii123

27. Sasaki H, Zlatescu M, Betensky R, Johnk L, Cutone A, Cairncross J, Louis D (2002) Histopathological-molecular genetic correlations in referral pathologist-diagnosed low-grade “oligodendroglioma”. J Neuropathol Exp Neurol 61:58-63

28. Schiffer D, Cavalla P, Migheli A, Chio A, Giordana M, Marino S, Attanasio A (1995) Apoptosis and cell proliferation in human neuroepithelial tumors. Neurosci Lett 195:81-84

29. Schiffer D, Dutto A, Cavalla P, Chio A, Migheli A, Piva R (1997) Role of apoptosis in the prognosis of oligodendrogliomas. Neurochem Int 31:245-250

30. Schrader C, Janssen D, Klapper W, Siebmann J, Meusers P, Brittinger G, Kneba M, Tiemann M, Parwaresch R (2005) Minichromosome maintenance protein 6 , a proliferation marker superior to Ki67 and independent predictor of survival in patients with mantle cell lymphoma. Br J Cancer 93:939-945

31. Scott I, Morris L, Rushbrook S, Bird K, Vowler S, Burnett N, Coleman N (2005) Immunohistochemical estimation of cell cycle entry and phase distribution in astrocytomas: applications in diagnostic neuropathology. Neuropathol Appl Neurobiol 31:455-466

32. Shetty A, Loddo M, Fanshawe T, Prevost A, Sainsbury R, Williams G, Stoeber K (2005) DNA replication licensing and cell cycle kinetics of normal and neoplastic breast. Br J Cancer 93:1295-1300

33. Smith J, Alderete B, Minn Y, Borell T, Perry A, Mohapatra G, Hosek S, Kimmel D, O'Fallon J, Yates A, Feuerstein B, 
Burger P, Scheithauer B, Jenkins R (1999) Localization of common deletion regions on $1 \mathrm{p}$ and $19 \mathrm{q}$ in human gliomas and their association with histological subtype. Oncogene 18:4144-4152

34. Smith J, Perry A, Borell T, Lee H, O'Fallon J, Hosek S, Kimmel D, Yates A, Burger P, Scheithauer B, Jenkins R (2000) Alterations of chromosome arms $1 p$ and $19 q$ as predictors of survival in oligodendrogliomas, astrocytomas, and mixed oligoastrocytomas. J Clin Oncol 18:636-645

35. Steel G (1977) Basic theory of growing cell populations. In: Steel GG (ed) Growth kinetics of tumours. Clarendon Press, Oxford, pp 56-85

36. Steinbach J, Weller M (2004) Apoptosis in gliomas: molecular mechanisms and therapeutic implications. J NeuroOncol 70:245-254

37. Stoeber K, Halsall I, Freeman A, Swinn R, Doble A, Morris L, Coleman N, Bullock N, Laskey R, Hales C, Williams G (1999) Immunoassay for urothelial cancers that detects DNA replication protein Mcm5 in urine. Lancet 354:1524-1525

38. Stoeber K, Tlsty T, Happerfield L, Thomas G, Romanov S, Bobrow L, Williams E, Williams G (2001) DNA replication licensing and human cell proliferation. J Cell Sci 114:2027-2041

39. Tada S, Li A, Maiorano D, Mechali M, Blow J (2001) Repression of origin assembly in metaphase depends on inhibition of RLF-B/Cdt1 by geminin. Nat Cell Biol 3:107-113

40. Takeda D, Dutta A (2005) DNA replication and progression through S phase. Oncogene 24:2827-2843

41. Van Den Bent M, Carpentier A, Brandes A, Sanson M, Taphoorn M, Bernsen H, Frenay M, Tijssen C, Grisold W, Sipos L, Haaxma-Reiche H, Kros J, Van Kouwenhoven M, Vecht C, Allgeier A, Lacombe D, Gorlia T (2006) Adjuvant procarbazine, lomustine and vincristine improves progres- sion-free survival but not overall survival in newly diagnosed anaplastic oligodendrogliomas and oligoastrocytomas: a randomized European organisation for research and treatment of cancer phase III trial. J Clin Oncol 24:2715-2711

42. Watanabe T, Nakamura M, Kros J, Burkhard C, Yonekawa Y, Kleihues P, Ohgaki H (2002) Phenotype vesus genotype correlation in oligodendrogliomas and low-grade diffuse astrocytomas. Acta Neuropathol 103:267-275

43. Wharton S, Hamilton F, Chan W, Chan K, Anderson J (1998) Proliferation and cell death in oligodendrogliomas. Neuropathol Appl Neurobiol 24:21-28

44. Wharton S, Chan K, Anderson J, Stoeber K, Williams G (2001) Replicative $\mathrm{Mcm} 2$ protein as a novel proliferation marker in oligodendrogliomas and its relationship to Ki67 labelling index, histological grade and prognosis. Neuropathol Appl Neurobiol 27:305-313

45. Wharton S, Hibberd S, Eward K, Crimmins D, Jellinek D, Levy D, Stoeber K, Williams G (2004) DNA replication licensing and cell cycle kinetics of oligodendroglial tumours. Br J Cancer 91:262-269

46. Williams G, Romanowski P, Morris L, Madine M, Mills A, Stoeber K, Marr J, Laskey R, Coleman N (1998) Improved cervical smear assessment using antibodies against proteins that regulate DNA replication. Proc Natl Acad Sci 95:1493214937

47. Williams G, Swinn R, Prevost A, De Clive-Lowe P, Halsall I, Going J, Hales C, Stoeber K, Middleton S (2004) Diagnosis of oesophageal cancer by detection of minichromosome maintenance 5 protein in gastric aspirates. Br J Cancer 91:714-719

48. Wohlshlegel J, Dwyer B, Dhar S, Cvetic C, Walter J, Dutta A (2000) Inhibition of eukaryotic DNA replication by geminin binding to Cdt1. Science 290:2271-2273 